\title{
Impact of DECISION + 2 on patient and physician assessment of shared decision making implementation in the context of antibiotics use for acute respiratory infections
}

France Légaré ${ }^{1,2^{*}}$, Mireille Guerrier ${ }^{1}$, Catherine Nadeau' ${ }^{1}$ Caroline Rhéaume ${ }^{2,3}$, Stéphane Turcotte ${ }^{1}$ and Michel Labrecque $e^{1,2}$

\begin{abstract}
Background: DECISION + 2, a training program for physicians, is designed to implement shared decision making (SDM) in the context of antibiotics use for acute respiratory tract infections (ARTIs). We evaluated the impact of DECISION + 2 on SDM implementation as assessed by patients and physicians, and on physicians' intention to engage in SDM.

Methods: From 2010 to 2011, a multi-center, two-arm, parallel randomized clustered trial appraised the effects of DECISION +2 on the decision to use antibiotics for patients consulting for ARTIs. We randomized 12 family practice teaching units (FPTUs) to either DECISION + 2 or usual care. After the consultation, both physicians and patients independently completed questionnaires based on the D-Option scale regarding SDM behaviors during the consultation. Patients also answered items assessing the role they assumed during the consultation (active/collaborative/passive). Before and after the intervention, physicians completed a questionnaire based on the Theory of Planned Behavior to measure their intention to engage in SDM. To account for the cluster design, we used generalized estimating equations and generalized linear mixed models to assess the impact of DECISION +2 on the outcomes of interest.
\end{abstract}

Results: A total of 270 physicians (66\% women) participated in the study. After DECISION +2, patients' D-Option scores were $80.1 \pm 1.1$ out of 100 in the intervention group and $74.9 \pm 1.1$ in the control group $(p=0.001)$. Physicians' $D$-Option scores were $79.7 \pm 1.8$ in the intervention group and $76.3 \pm 1.9$ in the control group $(p=0.2)$. However, subgroup analyses showed that teacher physicians D-Option scores were $79.7 \pm 1.5$ and $73.0 \pm 1.4$ respectively $(p=0.001)$. More patients reported assuming an active or collaborative role in the intervention group (67.1\%), than in the control group (49.2\%) $(p=0.04)$. There was a significant relation between patients' and physicians' D-Option scores $(p<0.01)$ and also between patient-reported assumed roles and both D-Option scores (as assessed by patients, $p<0.01$; and physicians, $p=0.01$ ). DECISION +2 had no impact on the intention of physicians to engage in SDM.

Conclusion: DECISION + 2 positively influenced SDM behaviors as assessed by patients and teacher physicians. Physicians' intention to engage in SDM was not affected by DECISION + 2.

Trial registration: ClinicalTrials.gov trials register no. NCT01116076.

Keywords: Shared decision making, Implementation, Theory of planned behavior, Training

\footnotetext{
* Correspondence: France.legare@mfa.ulaval.ca

${ }^{1}$ Research Center of the Centre Hospitalier Universitaire de Québec, Hôpital

St-François d'Assise, 10, Rue Espinay, Quebec City, QC G1L 3 L5, Canada

${ }^{2}$ Department of Family Medicine and Emergency Medicine, Université Laval,

Quebec City, QC, Canada

Full list of author information is available at the end of the article
} 


\section{Background}

Despite recent efforts to decrease prescription rates, antibiotics are still too frequently prescribed for acute respiratory tract infections (ARTIs) [1]. Results of attempts to improve the clinical decision making process regarding the use of antibiotics for ARTIs have been weakly conclusive, and interventions to reduce the use of antibiotics have mostly shown only modest improvements [2,3]. Scientific uncertainty about use of antibiotics as well as a failure to take into account the perspectives of both parties (patient as well as health professional), each with their own kind of expertise, may explain these results. In this clinical context, shared decision making (SDM) is an interesting pathway in the pursuit of optimal decisions. DECISION +2 , a training program for physicians, is designed to implement SDM in the context of antibiotics use for ARTIs $[4,5]$.

Engaging in SDM consists of encouraging patients to participate in the decision making process while sharing and reviewing patient values and preferences and the relative importance of the benefits and risks associated with treatment options. SDM encompasses a series of steps, each of which can be referred to as a specific SDM behavior [6,7]. In the clinical decision making process, the patient may choose to assume a number of roles, ranging from fully autonomous (patient selects his/her own treatment alone) through truly collaborative (physician and patient share the decision) to passive (physician makes the decision alone or hardly takes the patient's view into account) [8]. The role the patient assumes that best matches the SDM paradigm is the collaborative role, though moving patients from a passive to a more active role may also be considered an important step toward SDM [9].

Few authors have studied the implementation of SDM in routine clinical settings, and the literature is not yet specific about the most effective types of intervention for increasing healthcare professionals' adoption of SDM behaviors [10]. While behavior change interventions are essential to improving the practice of clinical medicine, a thorough understanding of the mechanisms underlying SDM behaviors is necessary in order to implement such changes $[11,12]$. In response to the disappointing results of many implementation interventions, researchers are increasingly using socio-cognitive theories to increase our understanding of underlying behavior mechanisms that might inform the design of further interventions [13]. The Theory of Planned Behavior (TPB) is one of the most frequently used theories for probing the factors that influence any individual behaviors, including those of patients and physicians [14-17]. It states that a specific behavior is mainly explained by the intention to perform it, and the intention itself can be predicted by measuring its three main determinants: attitude, subjective norm and perceived behavioral control [18]. In order to better understand the main results of this trial and better inform future SDM implementation studies, we deemed it essential to assess and reflect on the theoretical underpinnings of the DECISION + 2 intervention. Consequently, as a secondary objective embedded within the main cluster randomized trial (cRT), we used the TPB to evaluate the impact of DECISION + 2 on SDM implementation as assessed by patients and physicians, and on physicians' intention to engage in SDM.

\section{Methods}

\section{Study design}

The study consisted of a multi-center two-arm parallel CRT in three stages: the baseline data collection (physician and patient recruitment); the intervention (DECISION + 2 ); and post-intervention data collection (patient recruitment). We conducted the trial in a network of 12 family practices teaching units (FPTUs) in the Department of Family Medicine and Emergency Medicine at Université Laval, Quebec, Canada. The 12 FPTUs were randomly allocated to either an intervention group exposed to DECISION +2 or a control group with no intervention (usual care) [4]. The randomization was performed by a biostatistician using web-based software. Due to the nature of the trial, blinding was not possible.

\section{Participants and recruitment procedure}

All 12 FPTUs in the network of the Department of Family and Emergency Medicine at Université Laval were eligible to participate. All teacher family physicians, residents and nurse practitioners who provide care in the FPTU walk-in clinics were also eligible. Exclusion criteria included previous participation in the DECISION + pilot project and leave of absence during the study duration. We included patients (adults or children accompanied by a parent or legal guardian) with a diagnosis of acute respiratory infection (bronchitis, otitis media, pharyngitis or rhinosinusitis) and for whom the use of antibiotics was subsequently considered either by the patient or the physician during the visit [5]. Patients in the baseline data collection were different from those in the post-intervention data collection; however the physicians were the same in both data collection periods. All participants signed an informed consent form approved by the review boards of the two health and social services centres involved, namely the Centre de santé et de Services Sociaux de la Vieille-Capitale and the Centre de Santé et de Services Sociaux du Nord de Lanaudière.

\section{Intervention group}

The DECISION + 2 training program includes a web-based self-tutorial, a face-to-face, interactive session using videos, exercises and decision support tools, and a reminder at the point of care. The program was adapted from the original 
DECISION + pilot training program for the purpose of the present study $[19,20]$. The web-based self-tutorial lasts about 120 minutes and was intended to develop knowledge and skills regarding the clinical decision making process concerning antibiotic treatments for ARTIs in primary care, including knowledge of: the probabilistic nature of a diagnosis of a bacterial versus a viral infection; scientific evidence regarding the risk/ benefit ratios of the options; communication techniques; and strategies to foster patients' participation in the decision making process [21]. Participants in face-toface small group interactive sessions reviewed the key components of the web-based self-tutorial to enhance their ability to integrate the process of SDM into their practice regarding the use of antibiotics for ARTIs. Face-to-face small group interactive sessions were given by a principal investigator of the study or by a teaching physician from FPTUs who had been trained beforehand.

\section{Control group}

Physicians and patients in the control group were not exposed to DECISION + 2. Physicians were instructed to provide usual care to eligible patients.

\section{Data collection and variables}

All participants completed self-administered questionnaires before and after DECISION +2 between July 2010 and April 2011. The questionnaire flowchart is presented in Figure 1. We assessed SDM behaviors after the clinical

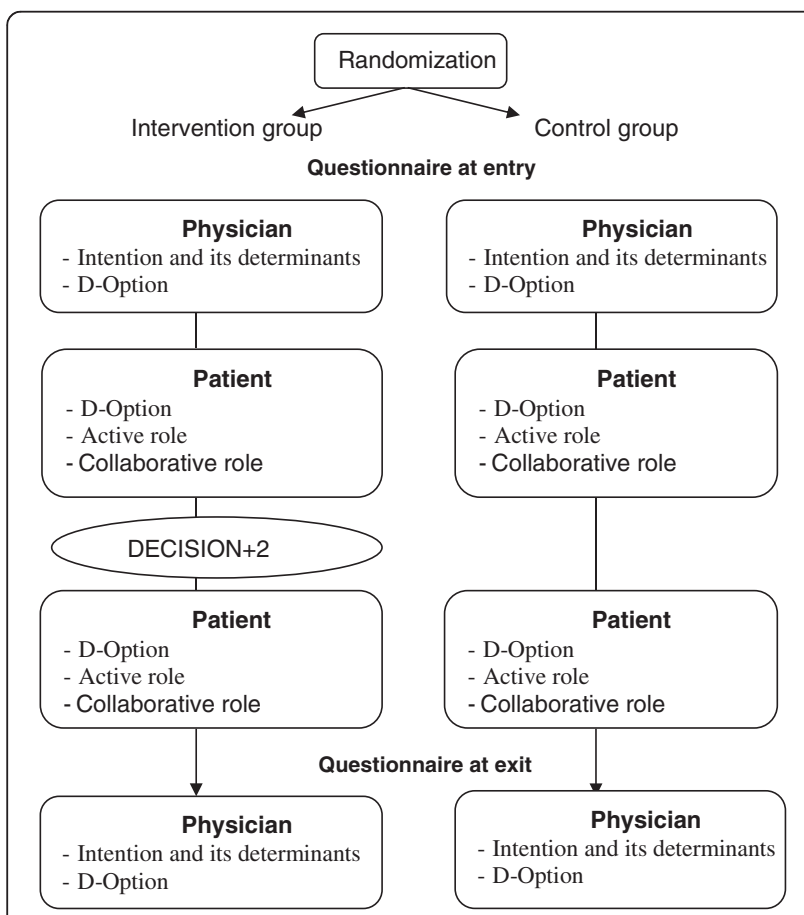

Figure 1 The questionnaire flowchart showing the outcomes assessed at each point of time. encounter using the D-Option scale $[22,23]$ and a modified version of the Control Preferences Scale (CPS), which we referred to as 'assumed role' [8]. Using a TPB-based scale, we collected data from participating physicians' intentions to engage in SDM and its related determinants (perceived behavioral control, subjective norm and attitude) at study entry and exit.

The D-Option is a scale used to assess perceived involvement in decision making from the perspective of both the patient and the physician (as opposed to that of a third observer) and includes 10 items. Both patients and their physicians independently completed the D-Option questionnaire after the consultation. We used mean scores out of a total of 100 . The modified CPS measures the patient's assumed role in decision making on a 1 to 5 response scale: 1 ) 'I made the decision'; 2) 'I made the decision after seriously considering my physician's opinion'; 3) 'My physician and I shared the responsibility for the decision making'; 4) 'My physician made the decision after seriously considering my opinion'; 5) 'My physician made the decision.' The patient playing an active role is indicated by responses 1 and 2, a collaborative role by 3 , and a passive role by 4 and 5 . For this study, we coded a new binary variable to combine the collaborative role and the active role (assumed to be SDM behaviors) as distinct from the passive role. Based on the $\mathrm{TPB}$, we assessed physicians' intention to engage in SDM in the future and its four related determinants: instrumental attitude and affective attitude were assessed by means of three items each, subjective norm by three items, and perceived behavioral control by three items. We used a 7point Likert scale from -3 (very low) to +3 (very high) to measure the intention and its related determinants. The reliabilities of the scales for behavioral intention and its determinants (instrumental attitude, affective attitude, subjective norm, and perceived behavioral control) were from 0.67 to 0.93 . We also collected sociodemographic characteristics of all participating physicians and patients.

\section{Statistical analysis}

We used descriptive statistics to describe characteristics of FPTUs, physicians, their scores of intention (and its determinants) and SDM behaviors. To take the nonindependence in the data (clustering effect) into account, we assessed the impact of the intervention on intention (and its determinants) using generalized linear mixed models (GLMMs) and assessed its impact on SDM behaviors using GLMMs for the D-Option scales and generalized estimating equations (GEE) for the patient's assumed role. We also used GEE and GLMMs to assess the relation among the three SDM behavior measures and to assess the relation between SDM behaviors and physician intention. We performed statistical analysis using the SAS version 9.3 (SAS Institute Inc., Cary, N.C., USA). 


\section{Results}

Flow of the trial and characteristics of participants

Figure 2 depicts the study flow. Out of the 12 eligible FPTUs, nine participated in the study: four in the control group and five in the intervention group. A total of 108 (68\% women) physicians and 162 (63\% women) physicians completed the questionnaire in the control and intervention groups respectively. However, we included only the participating physicians who had completed their entry questionnaire before DECISION + 2 was delivered. Thus we included 151 physicians in the intervention group and 99 in the control group (see Figure 2). Participants' sociodemographic characteristics are reported in Table 1.

Impact of DECISION + 2 on SDM behaviors as assessed by patients and physicians

At study entry, SDM behaviors as reported by patients and physicians showed similar results (Table 2). At study exit, we observed higher scores of SDM behavior as assessed by both physicians and patients using the DOption in the intervention group than in the control group, but the difference was only significant for patients (Table 2). Significantly more patients reported assuming an active and collaborative role in the intervention group than in the control group (Table 2). Also, we observed that DECISION + 2 seems to have a greater impact on patients reporting that they assumed an 'active' role than a 'collaborative' role (data not shown).

Subgroup analysis on family medicine residents and teacher physician groups revealed that patients reported a higher impact of DECISION + 2 on SDM behavior assessed
Table 1 Characteristics of participating FPTUs ${ }^{1}$ and physicians according to study groups

\begin{tabular}{lcc}
\hline & \multicolumn{2}{c}{$\mathbf{n} / \mathbf{N}(\%)$ of physicians $^{\mathbf{2}}$} \\
\cline { 2 - 3 } & Control & Intervention \\
\hline FPTUs $^{1}$ & $(n=4)$ & $(n=5)$ \\
Physicians & $(n=108)$ & $(n=162)$
\end{tabular}

Participating teachers

Female $\quad 36 / 53(68) \quad$ 49/78 (63)

Age, year, mean $\pm S D \quad 43.7 \pm 10 \quad 42.0 \pm 9.4$

Number of years in practice, mean \pm SD $\quad 15.2 \pm 10.7 \quad 13.9 \pm 10.3$

Residents

Female $\quad 34 / 55(62) \quad 60 / 84(72)$

$\begin{array}{lll}\text { Age, year, mean } \pm S D & 27.3 \pm 4.1 & 27.9 \pm 4.5\end{array}$

${ }^{1}$ FPTUs = Family practice teaching units.

${ }^{2}$ Unless otherwise indicated.

using D-Option with teacher physicians (intervention group: $79.7 \pm 1.5$ vs. control group: $73.0 \pm 1.4 ; \mathrm{p}=0.001$ ) than with residents (intervention group: $79.9 \pm 1.4$ vs. control group: $77.4 \pm 1.5 ; \mathrm{p}=0.21$ ). We observed no other statistically different impact in the subgroup analysis, and the results were similar to those for all the participants (i.e., residents and teacher physicians).

\section{Impact of DECISION + 2 on the intention to engage in SDM in the future}

Results of intention and its related determinants before and after the intervention are shown in Table 3. The mean scores of all TPB-based determinants of intention were high (there were no negative scores). We found no

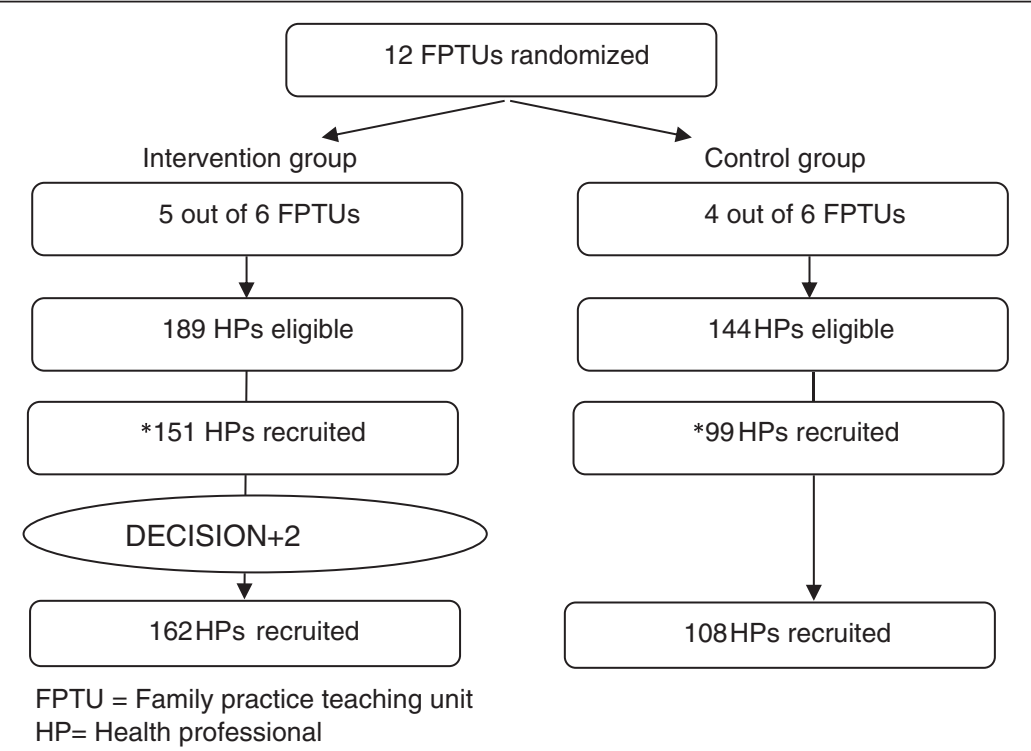

Figure 2 The flow study of participants showing the number of eligible health professionals in each group. *We included only the participating physicians who had completed their entry questionnaire before DECISION+2 was delivered. Thus we included 151 physicians in the intervention group and 99 in the control group. 
Table 2 Shared decision making behaviors at entry and exit in the study according to study groups

\begin{tabular}{|c|c|c|c|c|c|}
\hline \multirow[t]{2}{*}{ Behaviors } & \multicolumn{2}{|c|}{ Entry } & \multicolumn{2}{|c|}{ Exit } & \multirow[t]{2}{*}{$p$-value } \\
\hline & Control & Intervention & Control & Intervention & \\
\hline D-Option (Patient) & $80.0 \pm 1.5$ & $79.3 \pm 1.4$ & $74.9 \pm 1.1$ & $80.1 \pm 1.1$ & $0.001^{1}$ \\
\hline \multicolumn{6}{|l|}{ Mean \pm SD } \\
\hline D-Option (Physician) & $75.5 \pm 1.7$ & $74.4 \pm 2.1$ & $76.3 \pm 1.9$ & $79.7 \pm 1.8$ & 0.20 \\
\hline \multicolumn{6}{|l|}{ Mean \pm SD } \\
\hline Assumed role (patient) & & & & & $0.04^{2}$ \\
\hline Active/collaborative role $\mathrm{n}(\%)$ & $99(57.9)$ & $101(55.5)$ & $87(49.2)$ & $118(67.1)$ & \\
\hline Passive role $\mathrm{n}(\%)$ & $72(42.1)$ & $81(44.5)$ & $90(50.8)$ & $58(32.9)$ & \\
\hline
\end{tabular}

${ }^{1}$ Difference between groups at study exit evaluated with generalized linear mixed models to adjust for cluster design.

${ }^{2}$ Difference between groups at study exit evaluated with generalized estimating equations to adjust for cluster design.

significant difference in mean scores of the determinants of intention between the control group and the intervention group after DECISION +2 .

\section{Relation between SDM behaviors}

Table 4 details the relation between each possible pair of SDM behaviors. There was a statistically significant association between the SDM behaviors as assessed separately by patients and physicians using the D-Option scale (regression coefficient $=0.2, \mathrm{p}<0.01$ ). Furthermore, we observed a statistically significant association between the two D-Option scores and the patient-reported assumed role (Physician D-Option: regression coefficient $=3.4$, $\mathrm{p}=0.01$; Patient D-Option: regression coefficient $=6.8$, $\mathrm{p}<0.01)$.

Relation between behavioral intention and SDM behaviors Table 5 details the relation between physicians' intention to engage in SDM and each of the measures of SDM behaviors. We observed statistically significant association between physicians' perceived involvement in SDM, or D-Option (physician) and their intention to engage in SDM at study entry (regression coefficient $=3.5, \mathrm{p}<0.01$ ) and at exit (regression coefficient $=4.3, \mathrm{p}<0.01$ ). However, there was no association between physicians' intention and their SDM behaviors as assessed by patients, whether using D-Option (patient) or the modified CPS. Subgroup analysis for residents and teacher physicians did not differ statistically between residents and teacher physicians and were similar to the results obtained for both groups combined.

\section{Discussion}

Our study demonstrated a favorable impact of DECISION + 2 on SDM implementation in clinical practice as assessed by patients and teacher physicians using three measures. To the best of our knowledge, this full cRT is among the first to assess an SDM implementation intervention using the TPB. For example, none of the five trials that were reviewed for the Cochrane Collaboration included the use of the TPB in their trial [10]. In our study, three measures showed a statistically significant positive difference: patient-perceived involvement in SDM regarding the use of antibiotics for ARTIs as measured by D-Option (patient) and D-Option (teacher physician) and patient-reported assumed role in decision making as measured by the modified CPS. There was also a significant association between patient-perceived involvement in SDM and physician-perceived involvement (both DOption measures), as well as a significant association between patient-reported assumed role (modified CPS) and both patient- and physician-perceived involvement in SDM (using D-Option measures). However, our findings indicate that DECISION +2 had no impact on the

Table 3 Intention to engage in shared decision making and its related determinants at study entry and exit according to study groups

\begin{tabular}{|c|c|c|c|c|c|c|}
\hline \multirow[t]{3}{*}{ TPB constructs } & \multicolumn{2}{|c|}{ Entry } & \multicolumn{2}{|c|}{ Exit } & \multirow{3}{*}{$\begin{array}{c}\text { Mean } \\
\text { difference }^{1}\end{array}$} & \multirow[t]{3}{*}{$p$-value $^{1}$} \\
\hline & Control & Intervention & Control & Intervention & & \\
\hline & Mean \pm SD & Mean \pm SD & Mean \pm SD & Mean \pm SD & & \\
\hline Intention & $1.5 \pm 0.1$ & $1.6 \pm 0.1$ & $1.8 \pm 0.1$ & $1.7 \pm 0.1$ & 0.1 & 0.74 \\
\hline Instrumental attitude & $1.9 \pm 0.1$ & $1.9 \pm 0.1$ & $2.2 \pm 0.1$ & $2.2 \pm 0.1$ & 0 & 0.97 \\
\hline Affective attitude & $1.1 \pm 0.2$ & $1.3 \pm 0.1$ & $1.4 \pm 0.1$ & $1.6 \pm 0.1$ & 0.2 & 0.19 \\
\hline Subjective norm & $1.4 \pm 0.1$ & $1.5 \pm 0.1$ & $1.7 \pm 0.1$ & $1.6 \pm 0.1$ & 0.1 & 0.55 \\
\hline Perceived behavioral control & $1.1 \pm 0.1$ & $1.2 \pm 0.1$ & $1.3 \pm 0.1$ & $1.3 \pm 0.1$ & 0 & 0.99 \\
\hline
\end{tabular}

${ }^{1}$ Difference between groups at study exit evaluated with generalized linear mixed models to adjust for cluster design. 
Table 4 Relation between shared decision making behaviors

Shared decision making behaviors

\begin{tabular}{|c|c|c|c|c|}
\hline & \multicolumn{2}{|c|}{ D-Option (patient) } & \multicolumn{2}{|c|}{ D-Option (Physician) } \\
\hline & $\begin{array}{l}\text { Regression coefficient } \pm \\
\text { standard error }\end{array}$ & $p$-value & $\begin{array}{l}\text { Regression coefficient } \pm \\
\text { standard error }\end{array}$ & $p$-value \\
\hline D-Option (physician) & $0.2 \pm 0.1^{1}$ & $<0.01^{2}$ & - & - \\
\hline Assumed role (patient) (Active or collaborative vs. passive role) & $6.8 \pm 1.4^{3}$ & $<0.01^{4}$ & $3.4 \pm 1.2^{3}$ & $0.01^{4}$ \\
\hline
\end{tabular}

${ }^{1}$ Increase in the average value of D-Option (Patient) when the variable D-Option (Physician) increased by one point (out of 100).

${ }^{2}$ Evaluated with generalized linear mixed models to adjust for cluster design.

${ }^{3}$ Increase in the average value of D-Option (out of 100) for a patient who reported an active or collaborative role compared to a patient who reported a passive role.

${ }^{4}$ Evaluated with generalized estimating equations to adjust for cluster design.

intention of physicians to engage in SDM in the future, even though this intention was slightly associated with SDM behavior as assessed by the physician, or D-Option (physician). The results of a secondary analysis of the DECISION +2 study has led us to reflect further on the theoretical underpinnings of the DECISION +2 intervention, and this has increased our understanding of trial results published previously [5]. We therefore suggest that in all future SDM implementation studies, the theoretical underpinnings of the intervention implementation should be described and analyzed for better understanding of results. These results lead us to make four main observations.

First, our results show that it is possible to train physicians to encourage patients to assume a more active role in decision making about their care. In recent years, many healthcare systems have encouraged and empowered patients to be more involved in decision making $[24,25]$. These findings are an important achievement since many studies have reported that patient involvement in their care has a positive relationship to health status outcomes [26-29]. Our findings suggest that DECISION +2 has the potential to provide physicians with the skills and competencies needed for SDM to occur during consultation, and that both patients and physicians are responsive to the translation of these newly acquired skills and competencies into clinical practice.
Second, it is interesting to note that patients' and physicians' D-Option scores were slightly associated. Although there might be differences in what each party (patient/ physician) perceives as SDM behaviors, this association suggests that we should not dismiss tools that measure SDM behaviors from the perspective of both patients and physicians. Our findings suggest that the D-Option assessments (by patients and physicians separately) might provide a valuable measure of SDM behaviors. Our study also shows that SDM behaviors as measured using D-Option from each perspective (patient and physician) are associated with the role assumed by patients as measured using the modified version of the CPS. This provides valuable evidence of the 'measurement validity' of both D-Option and assumed roles as measured by the modified CPS. Interestingly, subgroup analysis showed that D-Option assessment by patients revealed that DECI$\mathrm{SION}+2$ had a significant impact on teacher physicians, as observed for the combined groups, but not on the residents, meaning that the impact of DECISION +2 on the combined groups was greatly influenced by its impact on the teacher physician subgroup. While this result can be interpreted in several different ways, the most likely explanation is that D-Option scores in the resident subgroup were higher than D-Option scores in the teacher physician subgroup. This difference with the control group

Table 5 Relation between shared decision making behaviors and physician intention

\begin{tabular}{|c|c|c|c|c|c|c|}
\hline \multicolumn{7}{|c|}{ Shared decision making behaviors } \\
\hline \multirow[b]{2}{*}{ Intention (physician) } & \multicolumn{2}{|c|}{ D-Option (patient) } & \multicolumn{2}{|c|}{ D-Option (physician) } & \multicolumn{2}{|c|}{$\begin{array}{l}\text { Assumed role (patient) (Active } \\
\text { or collaborative vs. passive role) }\end{array}$} \\
\hline & $\begin{array}{l}\text { Regression coefficient } \pm \\
\text { standard error }\end{array}$ & $p$-value & $\begin{array}{l}\text { Regression coefficient } \pm \\
\text { standard error }\end{array}$ & $p$-value & $\begin{array}{l}\text { Regression coefficient } \pm \\
\text { standard error }\end{array}$ & $p$-value \\
\hline Entry & $-0.1 \pm 0.9^{1}$ & $0.89^{2}$ & $3.5 \pm 0.7^{1}$ & $<0.01^{2}$ & $0.0 \pm 0.1^{3}$ & $0.81^{4}$ \\
\hline Exit & $0.5 \pm 1.0^{1}$ & $0.60^{2}$ & $4.3 \pm 0.8^{1}$ & $<0.01^{2}$ & $0.1 \pm 0.2^{3}$ & $0.60^{4}$ \\
\hline
\end{tabular}

${ }^{1}$ Increase in the average value of D-Option when the variable intention (physician) increased by one point (on a scale of -3 to +3 ).

${ }^{2}$ Evaluated with generalized linear mixed models to adjust for cluster design.

${ }^{3}$ With every increase of one point on the intention scale, the chances of a patient being active or collaborative rather than passive increased by e regression coefficient ( $0 \%$ at entry and $11 \%$ at exit).

${ }^{4}$ Evaluated with generalized estimating equations to adjust for cluster design. 
may have been caused by the fact that residents are more aware of new practice methods such as SDM and are also more likely to be sensitive to the on-going evaluation they are experiencing when training in the FPTUs.

Third, in considering descriptive statistics of patientreported assumed role (data not shown), we observed that DECISION + 2 seems to have a greater impact on patients reporting that they assumed an 'active' role than a 'collaborative' role. This difference may reflect a difference, from the patients' point of view, between being involved in the decision making and sharing it equally. Authors of an earlier study have pointed out that patients seem to distinguish between the process of involvement and who makes the final decision [30]. Another study found that patients who opted against cancer screening after reviewing a brochure or decision aid were less likely to discuss their decision with their physician; i.e., from the patients' point of view, they may be active in the decision without requiring any participation by the physician at all [31]. Therefore, it may be useful in future SDM implementation work to better distinguish between measuring the extent to which the patient engages in decision making (or moves towards a more active role in decision making) and the occurrence of SDM (where patient and physician make the decision together). As mentioned earlier, the 'active role' appears better aligned with an informed decision than a shared decision. Shared decision making refers to the middle ground between an informed decision making process and a paternalistic decision making process [32]. As demonstrated by the extensive use of the Control Preference Scale and Assumed Role in SDM studies [33], favouring an SDM process may lead to setting an optimal level of autonomy in patients so that they are never at the extreme end of the continuum: i.e., they never report having made the decision alone. Future research on the conceptualization of SDM should focus on this.

Fourth, we did not see any impact of DECISION +2 on the intention of physicians to engage in SDM in the future or on its determinants as defined by the TPB. The following points may provide an explanation. First, there was a ceiling effect, with physicians showing high scores on all these measures before the intervention. Since patient and public involvement is getting popular and physicians are more aware of SDM, it is not surprising that we observed high intention, as other studies have done $[17,34]$. Second, the TPB defines and distinguishes between several precise behaviors in great detail [35]. In our study, SDM behaviors were defined broadly, while engaging in SDM in fact encompasses many competencies, or more specifically a set of SDM behaviors. On the other hand, SDM behaviors as assessed by physicians after consultations with single patients were shown to be associated with their intention to engage in SDM at study entry and exit, thus providing some grounds for anticipating that this link between intention and behaviour will continue to be trustworthy in the future [14-17]. Lastly, in the case of highly recommended or desirable behaviors with high ceiling effects, the TPB may not be adequate for understanding behavior change.

This study has a number of limitations. First, it was embedded in a larger study and was not designed nor powered for our stated objective. Second, our results regarding patient involvement in decision making could have been overestimated due to the use of patient and physician self-reported measures, a problem that could be overcome by using an objective measurement such as third-observer instrument (e.g., the OPTION observer instrument). Third, physicians practicing in FPTUs are not representative of the whole population of physicians and may be more aware of SDM since they are immersed in an academic environment where SDM and patient involvement is valued throughout the curriculum. Physicians who participated may be more interested in SDM, which may have led to a weak overestimation of the impact of DECISION +2 on the entire physician population (ceiling effect). However, due to the high rate of participation, the impact should be negligible. We did not expect much contamination between groups because physicians needed a personal access code to the web-based tutorial and we also made sure to take attendance during the live workshop. Finally, as discussed above, we can presume that a desirability bias occurred because of the increasing popularity of and public pressure for patient and public involvement programs, as suggested by the high scores of intention and its determinants such as attitude $[34,36]$.

\section{Conclusions}

Our findings suggest that although engaging in SDM may be a complex behavior for physicians to adopt, DECISION +2 , a training program, has the potential to provide physicians with the skills and competencies needed for the successful implementation of SDM in clinical practice. The results also indicate that both patients and physicians are responsive to the translation of these newly acquired skills and competencies into their behaviour during consultations, and that both parties share some common understanding of SDM behaviors.

\section{Abbreviations}

ARTIs: Acute respiratory treat infections; CPS: Control preference scale; CRT: Cluster randomized trial; FPTUs: Family practice teaching units; SDM: Shared decision making; TPB: Theory of planned behavior.

\section{Competing interests}

The authors declare that they have no competing interests.

\section{Authors' contributions}

All authors made substantial contributions to the interpretation of the data. $\mathrm{ML}$ and FL planned and coordinated the study. FL, MG and CN wrote the first draft of the manuscript. ST and MG analyzed the data and performed statistical analyses. All authors reviewed and approved the final manuscript. 


\section{Acknowledgments}

This study is supported by a grant from the Institut National d'Excellence en Santé et en Services Sociaux (INESS)/ Fonds de la recherche du QuébecSanté and was accepted by the research ethic board of Centre des Services Sociaux de la Vieille Capitale. FL holds the Canada Research Chair in Implementation of Shared Decision Making in Primary Care. FL and ML are members of Knowledge Translation Canada. The authors would like to underline the contribution of the patients and physicians who participated in the study.

\section{Author details}

${ }^{1}$ Research Center of the Centre Hospitalier Universitaire de Québec, Hôpital St-François d'Assise, 10, Rue Espinay, Quebec City, QC G1L 3 L5, Canada. ${ }^{2}$ Department of Family Medicine and Emergency Medicine, Université Laval, Quebec City, QC, Canada. ${ }^{3}$ Research Center of Institut Universitaire de Cardiologie et de Pneumologie de Québec, Quebec City, QC, Canada.

Received: 7 June 2013 Accepted: 23 December 2013

Published: 26 December 2013

\section{References}

1. Patrick DM, Hutchinson J: Antibiotic use and population ecology: how you can reduce your "resistance footprint". Can Med Assoc J 2009, 180:416-421.

2. Arnold SR, Straus SE: Interventions to improve antibiotic prescribing practices in ambulatory care. Cochrane Database Syst Rev 2005, 4, CD003539.

3. Butler CC, Hood K, Verheij T, Little P, Melbye H, Nuttall J, Kelly MJ, Molstad S, Godycki-Cwirko M, Almirall J, et al: Variation in antibiotic prescribing and its impact on recovery in patients with acute cough in primary care: prospective study in 13 countries. BMJ 2009, 338:b2242.

4. Legare F, Labrecque M, Godin G, LeBlanc A, Laurier C, Grimshaw J, Castel J, Tremblay I, Fremont P, Cauchon M, et al: Training family physicians and residents in family medicine in shared decision making to improve clinical decisions regarding the use of antibiotics for acute respiratory infections: protocol for a clustered randomized controlled trial. BMC Fam Pract 2011, 12:3.

5. Legare F, Labrecque M, Cauchon M, Castel J, Turcotte S, Grimshaw J: Training family physicians in shared decision-making to reduce the overuse of antibiotics in acute respiratory infections: a cluster randomized trial. CMAJ 2012, 184(13):E726-E734.

6. Makoul G, Clayman ML: An integrative model of shared decision making in medical encounters. Patient Educ Couns 2006, 60(3):301-312.

7. Towle A, Godolphin W: Framework for teaching and learning informed shared decision making. BMJ 1999, 319(7212):766-771.

8. Degner LF, Sloan JA: Decision making during serious illness: what role do patients really want to play? J Clin Epidemiol 1992, 45(9):941-950.

9. Weston WW: Informed and shared decision-making: the crux of patientcentered care. CMAJ 2001, 165(4):438-439.

10. Legare F, Ratte S, Stacey D, Kryworuchko J, Gravel K, Graham ID, Turcotte S: Interventions for improving the adoption of shared decision making by healthcare professionals. Cochrane Database Syst Rev 2010, 5, CD006732.

11. Michie S, van Stralen MM, West R: The behaviour change wheel: a new method for characterising and designing behaviour change interventions. Implement Sci 2011, 6(1):42.

12. Legare F, Bekker H, Desroches S, Drolet R, Politi MC, Stacey D, Borduas F, Cheater FM, Cornuz J, Coutu MF, et al: How can continuing professional development better promote shared decision-making? Perspectives from an international collaboration. Implement Sci 2011, 6(1):68.

13. Ramsay CR, Thomas RE, Croal BL, Grimshaw JM, Eccles MP: Using the theory of planned behaviour as a process evaluation tool in randomised trials of knowledge translation strategies: a case study from UK primary care. Implement Sci 2010, 5:71.

14. Legare F, Graham ID, O'Connor AC, Aubin M, Baillargeon L, Leduc Y, Maziade J: Prediction of health professionals' intention to screen for decisional conflict in clinical practice. Health Expect 2007, 10(4):364-379.

15. Legare F, Borduas F, Jacques A, Laprise R, Voyer G, Boucher A, Luconi F, Rousseau M, Labrecque M, Sargeant J, et al: Developing a theory-based instrument to assess the impact of continuing professional development activities on clinical practice: a study protocol. Implement Sci 2011, 6:17.

16. Godin G, Belanger-Gravel A, Eccles M, Grimshaw J: Healthcare professionals' intentions and behaviours: a systematic review of studies based on social cognitive theories. Implement Sci 2008, 3:36.
17. Eccles MP, Francis J, Foy R, Johnston M, Bamford C, Grimshaw JM, Hughes J, Lecouturier J, Steen N, Whitty PM: Improving professional practice in the disclosure of a diagnosis of dementia: a modeling experiment to evaluate a theory-based intervention. Int J Behav Med 2009, 16(4):377-387.

18. Armitage CJ, Conner M: Efficacy of the theory of planned behaviour: a meta-analytic review. Br J Soc Psychol 2001, 40(Pt 4):471-499.

19. Allaire AS, Labrecque M, Giguere A, Gagnon MP, Grimshaw J, Legare F: Barriers and facilitators to the dissemination of DECISION+, a continuing medical education program for optimizing decisions about antibiotics for acute respiratory infections in primary care: a study protocol. Implement Sci 2011, 6(1):3.

20. Legare F, Labrecque M, Leblanc A, Njoya M, Laurier C, Cote L, et al: Training family physicians in shared decision making for the use of antibiotics for acute respiratory infections: a pilot clustered randomized controlled trial. Health Expect 2011, Suppl 1:96-110

21. Legare F, Labrecque M, Leblanc A, Thivierge R, Godin G, Laurier C, Cote L, O'Connor AM, Allain-Boule N, Rousseau J, et al: Does training family physicians in shared decision making promote optimal use of antibiotics for acute respiratory infections? Study protocol of a pilot clustered randomised controlled trial. BMC Fam Pract 2007, 8:65.

22. Melbourne E, Roberts S, Durand MA, Newcombe R, Legare F, Elwyn G: Dyadic OPTION: measuring perceptions of shared decision-making in practice. Patient Educ Couns 2011, 83(1):55-57.

23. Elwyn G, Edwards A, Wensing M, Hood K, Atwell C, Grol R: Shared decision making: developing the OPTION scale for measuring patient involvement. Qual Saf Health Care 2003, 12(2):93-99.

24. European Commission Directorate-General for health and Consumers: TNS Qual+. Eurobarometer Qualitative Study. PATIENT INVOLVEMENT. Aggregate Report. 2012 [cited 201324 December]. Available from: http://ec.europa.eu/ public_opinion/archives/quali/ql_5937_patient_en.pdf.

25. The Expert Patient: A New Approach to Chronic Disease Management for the 21st Century. [http://webarchive.nationalarchives.gov.uk/20130107105354/ http://www.dh.gov.uk/prod_consum_dh/groups/dh_digitalassets/@dh/@en/ documents/digitalasset/dh_4018578.pdf].

26. Hibbard JH, Greene J: What the evidence shows about patient activation: better health outcomes and care experiences; fewer data on costs. Health Aff (Millwood) 2013, 32(2):207-214.

27. Hibbard $J \mathrm{H}$, Greene J, Overton V: Patients with lower activation associated with higher costs; delivery systems should know their patients' 'scores'. Health Aff (Millwood) 2013, 32(2):216-222.

28. Thompson AG: The meaning of patient involvement and participation in health care consultations: a taxonomy. Soc Sci Med 2007, 64(6):1297-1310.

29. Elwyn G, Edwards A, Kinnersley P, Grol R: Shared decision making and the concept of equipoise: the competences of involving patients in healthcare choices. Br J Gen Pract 2000, 50(460):892-899.

30. Edwards A, Elwyn G: Inside the black box of shared decision making: distinguishing between the process of involvement and who makes the decision. Health Expect 2006, 9(4):307-320.

31. Frosch DL, Legare F, Mangione CM: Using decision aids in community-based primary care: a theory-driven evaluation with ethnically diverse patients. Patient Educ Couns 2008, 73(3):490-496.

32. Charles C, Gafni A, Whelan T: Shared decision-making in the medical encounter: what does it mean? (or it takes at least two to tango). Soc Sci Med 1997, 44(5):681-692

33. Legare F, Turcotte S, Stacey D, Ratte S, Kryworuchko J, Graham ID: Patients' perceptions of sharing in decisions: a systematic review of interventions to enhance shared decision making in routine clinical practice. Patient 2012, 5(1):1-19.

34. Legare F, St-Jacques S, Gagnon S, Njoya M, Brisson M, Fremont P, Rousseau $F$, et al: Prenatal screening for Down syndrome: a survey of willingness in women and family physicians to engage in shared decision-making. Prenat Diagn 2011, 31(4):319-326.

35. Ajzen I: The theory of planned behavior. Organ Behav Hum Decis Process 1991, 50(2):179-211.

36. Holmes-Rovner M, Gruman J, Rovner DR: Shared decision-making in the US-research \& development outpaces delivery. Z Arztl Fortbild Qualitatssich 2007, 101(4):254-258.

\section{doi:10.1186/1748-5908-8-144}

Cite this article as: Légaré et al:: Impact of DECISION + 2 on patient and physician assessment of shared decision making implementation in the context of antibiotics use for acute respiratory infections. Implementation Science 2013 8:144 